\title{
Behavioral Conformity in Games with Many Players
}

by

Myrna Wooders, Edward Cartwright, and Reinhard Selten

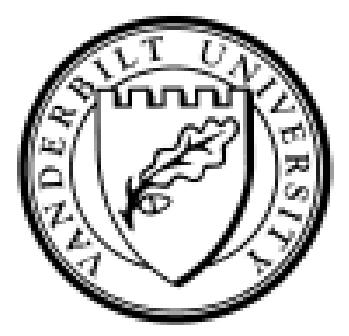

Working Paper No. 05-W13

April 2005

\section{DEPARTMENT OF ECONOMICS \\ VANDERBILT UNIVERSITY \\ NASHVILLE, TN 37235}

www.vanderbilt.edu/econ 


\title{
Behavioral Conformity in Games with Many Players*
}

\author{
Myrna Wooders ${ }^{\dagger}$ \\ Vanderbilt University and University of Warwick, \\ Departments of Economics \\ Coventry CV4 7AL, UK \\ M.Wooders@warwick.ac.uk \\ http://www.myrnawooders.com \\ Edward Cartwright \\ University of Kent, Department of Economics \\ Canterbury, \\ Kent.CT2 7NP. UK \\ e.cartwright@tiscali.co.uk \\ Reinhard Selten \\ University of Bonn, Department of Economics \\ Adenauerallee 24-26, 53113 Bonn \\ Germany
}

This version: March 2005

JEL Codes: C 72, Z 13

Keywords: behavioral conformity, noncooperative games, pregames, Nash equilibrium, purification, social norms, behavorial norms

\footnotetext{
${ }^{*}$ We are indebted to an Associate Editor of Games and Economic Behavior and a referee for their helpful comments; their intellectual generosity is greatly appreciated.

${ }^{\dagger}$ This author is indebted to Sonderforschungsbereich 303 and to the University of Bonn for hospitality and financial support during 1990-1991 when this research was initiated.
} 


\begin{abstract}
In the literature of psychology and economics it is frequently observed that individuals tend to conform in their behavior to the behavior of similar individuals. A fundamental question is whether the outcome of such behavior can be consistent with self-interest. We propose that this consistency requires the existence of a Nash or approximate Nash equilibrium that induces a partition of the player set into relatively few societies, each consisting of similar individuals playing the same or similar strategies. In this paper we introduce a notion of a society and characterize a family of games admitting the existence of such an equilibrium. We also introduce the concept of 'crowding types' into our description of players and distinguish between the crowding type of a player - those characteristics of a player that have direct effects on others- and his tastes, taken to directly affect only that player. With the assumptions of 'within crowding type anonymity' and 'linearity of taste-types' we show that the number of groups can be uniformly bounded.
\end{abstract}

\title{
1 Behavioral conformity
}

Individuals belonging to the same society typically have commonalities of language, social and behavioral norms, and customs. A fundamental question is whether behavioral conformity can be consistent with self-interested behavior. From the perspective of game theory, we propose that this consistency requires the existence of a Nash equilibrium or an approximate Nash equilibrium that induces a partition of players into societies where all individuals within the same society are similar and play the same or similar strategies and where most or all societies are nontrivial in size. The Nash equilibrium captures a notion of self-interested behavior while the existence of large societies facilitates conformity within societies. In this paper we introduce a notion of a society consisting of similar individuals and describe a family of games where an equilibrium with the desired properties exists. Two conformity results are established, one for approximate Nash equilibrium and another, with stronger conditions on the model, for exact equilibrium.

To address the question of whether conformity can be individually rational we introduce a structure generating games with the property that, if there are many players, for most players there are many similar players. It may seem intuitive that continuity properties, ensuring that players who have similar attributes are similar, would lead to the existence of Nash equi- 
librium where all players who are similar players choose similar or the same strategies. In fact, in general this is false. (See also Example 1.) To obtain behavioral conformity within societies, these must be carefully defined; in particular, players on the 'boundaries' of a society need not conform to the behavior of members of that society. Further, since it is difficult to motivate the use of mixed strategies if players imitate or conform, we require that all players belonging to a society to play the same pure strategy.

To obtain our results, we make two assumptions. The first ensures that players with similar attributes are indeed similar as players in induced games. The second ensures that the strategy choices of individual players have near-negligible impacts on other players. Our main result can be summarized:

Conformity: Given any $\varepsilon>0$ there are integers $\eta(\varepsilon)$ and $A(\varepsilon)$ such that any game with at least $\eta(\varepsilon)$ players has a Nash $\varepsilon$-equilibrium in pure strategies that induces a partition of the population into at most $A(\varepsilon)$ societies.

Note that the bound on the number of societies $A$ is independent of the size of the population. Thus, if there are 'many' players then most societies must be large. Moreover, the smaller the number of societies, the greater the possible difference between players in the same society and the stronger the conformity. Generally, however, the bound $A$ is not independent of $\varepsilon$. With an additional assumption, we also demonstrate:

Uniform boundedness of the number of societies: With 'linearity in taste types,' the bound on the number of societies $A$ is independent of $\varepsilon$.

We proceed as follows: Section 2 introduces notation and definitions. In Section 3 we treat conformity beginning with some simple examples before providing our two main results and a discussion on normative influence. In Section 4 we conclude and an Appendix contains remaining proofs.

\section{Notation and definitions}

A game $\Gamma$ is given by a triple $\left(N, S,\left\{u_{i}\right\}_{i \in N}\right)$ consisting of a finite player set $N$, a finite set of $K$ pure strategies $S=\left\{s_{1}, \ldots, s_{K}\right\}$, and a set of payoff functions $\left\{u_{i}\right\}_{i \in N}$. A pure strategy vector for game $\Gamma$ is given by $m=$ $\left(m_{1}, \ldots, m_{|N|}\right)$ where $m_{i} \in S$ denotes the pure strategy of player $i$. The set of pure strategy vectors is given by $S^{N}$. We note that for each $i \in N$ the payoff function $u_{i}$ maps $S^{N}$ into the real line. 
Let $\Omega$ be a compact metric space, called an attribute space, let $S$ be a finite set of strategies, and let $W$ be the set of all mappings from $\Omega \times S$ into $\mathbb{R}_{+}$with finite support, that is, $w \in W$ takes on nonzero values for at most a finite number of points in its domain. ${ }^{1}$ A member of $W$ is called a weight function. A non-cooperative pregame is a triple $\mathcal{G}=(\Omega, S, h)$ consisting of an attribute space $\Omega$, a set of pure strategies $S$ and a function $h: \Omega \times S \times W \longrightarrow$ $\mathbb{R}_{+}$. As we formalize below, the function $h$ determines a payoff function for each player in any game induced by a pregame; the payoff to a player depends on the attributes of that player, his strategy choice, and the weight function induced by the strategy choices of the other players.

Take as given a pregame $\mathcal{G}=(\Omega, S, h)$. Let $N=\{1, \ldots,|N|\}$ be a finite set and let $\alpha$ be a mapping from $N$ to $\Omega$, called an attribute function. The pair $(N, \alpha)$ is a population. In interpretation, $N$ will be a set of players and $\alpha$ provides a description of the players in terms of their attributes. A pure strategy vector for the population $(N, \alpha)$ is given by a vector $m=$ $\left(m_{1}, \ldots, m_{|N|}\right)$ where $m_{i} \in S$ ascribes a pure strategy to $i \in N$.

Given a population $(N, \alpha)$ and a pure strategy vector $m \in S^{N}$ we say that weight function $w_{\alpha, m} \in W$ is relative to $m$ if,

$$
w_{\alpha, m}\left(\omega, s_{k}\right)=\mid\left\{i \in N: \alpha(i)=\omega \text { and } m_{i}=s_{k}\right\} \mid
$$

for all $s_{k} \in S$ and all $\omega \in \Omega$. Thus, $w_{\alpha, m}\left(\omega, s_{k}\right)$ denotes the number of players with attribute $\omega$ who play strategy $s_{k}$. An induced game $\Gamma(N, \alpha)$ can now be defined:

$$
\Gamma(N, \alpha)=\left(N, S,\left\{u_{i}^{\alpha}: S^{N} \longrightarrow \mathbb{R}_{+}\right\}_{i \in N}\right)
$$

where

$$
u_{i}^{\alpha}(m) \stackrel{\text { def }}{=} h\left(\omega, m_{i}, w_{\alpha, m}\right)
$$

for all $\omega \in \alpha(N)$ and $m$. We note that players who are ascribed the same attribute have the same payoff function.

Other than finiteness of the strategy set, a pregame need not imply any assumptions on the induced games. A pregame, however, provides a useful framework in which (a) to treat a family of games all induced from a common strategic situation as given by the attribute space $\Omega$ and pure strategy set $S$, and (b) to be able, relatively simply, to impose assumptions on that family of games through the function $h$. We demonstrate this later point in Section 3.

\footnotetext{
${ }^{1}$ Where $\mathbb{R}_{+}$denotes the non-negative real numbers.
} 
We will assume throughout that players play pure strategies. We invoke, however, the standard von Neumann Morgenstern assumptions with regard to expected utility of (mixed) strategies. The standard definition of a Nash equilibrium applies. Given $\varepsilon \geq 0$, a strategy vector $m$ is a $N a s h \varepsilon$-equilibrium in pure strategies or, informally, an approximate Nash equilibrium in pure strategies, only if

$$
u_{i}^{\alpha}\left(m_{i}, m_{-i}\right) \geq u_{i}^{\alpha}\left(s_{k}, m_{-i}\right)-\varepsilon
$$

for all $i \in N$ and $s_{k} \in S$.

\subsection{Societies}

Throughout we assume, for convenience, a particular form of attribute space. Let $\mathcal{C}=\{1,2, \ldots, C\}$ be a finite set of crowding types. ${ }^{2}$ We assume that $\Omega$ is given by $\mathcal{C} \times[0,1]^{F}$ for some finite integer $F \geq 1 .^{3}$ We will typically denote an attribute by $\omega=(c, t)$ where $c \in \mathcal{C}$ and $t \in[0,1]^{F}$. We use the metric on $\Omega$ whereby the distance between two attributes $\omega=(c, t)$ and $\omega^{\prime}=\left(c^{\prime}, t^{\prime}\right)$ is 2 if $c \neq c^{\prime}$ and equals the $\max _{f}\left|t_{f}-t_{f}^{\prime}\right|$ otherwise. In interpretation, therefore, two players $i$ and $j$ with the same crowding type are always seen as 'more similar' than two players with different crowding types. The attribute space will be treated in more detail in Section 3.3 and 3.4 .

Given an attribute function $\alpha$ we define function $\tau: N \rightarrow[0,1]^{F}$ by $\tau(i)=t$ if and only if $\alpha(i)=(c, t)$ for some $c$. Thus, $\tau$ is the projection of $\alpha$ onto $[0,1]^{F}$. Given a set $A$ we denote by $\operatorname{con}(A)$ the convex hull of $A$ and by $\operatorname{int}(A)$ the interior of $A$.

A society: Given population $(N, \alpha)$ and strategy vector $m$ a set of players $D \subset N$ is a society (relative to $\alpha$ and $m$ ) if, for some crowding type $c$ the following three conditions are satisfied:

1. for all players $i, j \in D, m_{i}=m_{j}$ (all players in $D$ choose the same pure strategy),

2. for every player $i \in D, \alpha(i)=(c, t)$ for some $t$ (all players in $D$ have crowding type $c$ ),

\footnotetext{
${ }^{2}$ The term 'crowding type' is taken from Conley and Wooders (2001) and their earlier papers. Crowding types are described further in the next section, where they play a significant role.

${ }^{3}$ This appears more than general enough to cover many potential applications. Results for a more general form of attribute space are obtained by Wooders, Cartwright and Selten (2001).
} 
3. for any player $i \in N$, if $\alpha(i)=(c, t)$ and $t \in \operatorname{int}(\operatorname{con}(\tau(D))$ then $i \in D$.

We say that a pure strategy vector $m$ induces a partition of the population $(N, \alpha)$ into a set of societies $\mathcal{S}=\left\{N_{1}, \ldots, N_{Q}\right\}$ if each player $i \in N$ belongs to a unique society $N_{q} \in \mathcal{S}$ and if each society $N_{q} \in \mathcal{S}$ is relative to $\alpha$ and $m$. Note that if $m$ induces a partition of the population $(N, \alpha)$ into $Q$ societies then there exists a partition of $[0,1]^{F}$ into $Q$ convex subsets $\left\{T_{q}\right\}_{q=1}^{Q}$ such that, for any two players $i, j \in N$ and any $T_{q}$, if $\alpha(i)=(c, t), \alpha(j)=\left(c, t^{\prime}\right)$ and $\tau(i), \tau(j) \in \operatorname{int}\left(T_{q}\right)$ then $m_{i}=m_{j}$.

The definition of a society captures two key features. First, players in the same society play the same strategy; this is clearly motivated by the observation that 'social conformity' may lead to common behavior. Second, players in the same society have similar attributes; this is motivated by the observation that a player may only conform to those with whom he identifies. The second feature is captured by the requirement that all players in a society have the same crowding type and that all members of a society have attributes in the same convex subset of attribute space. Note also that there may well be 'marginal players', individuals with taste attributes on the boundary of those for some society and who play the same or a different strategy than the society members. Allowing such marginal players is essential for our conformity results,

We note that any strategy vector induces a partition of a population $(N, \alpha)$ into $|N|$ societies where each society consists of one player. ${ }^{4}$ A crucial aspect of our main results will thus be to bound the number of societies independently of the size of the player set. In games with many players, this will ensure that some societies contain many players. We treat other implications of the definition of a society after stating our main result in Section 3.3.

\section{Conformity}

Before introducing our results we provide two simple examples to show why an equilibrium with the desired properties may not exist.

Example 1: Players have to choose between two locations $A$ and $B$. The attribute space is given by $\{X, P\}$ where a player with crowding type $X$ is a celebrity and a player with crowding type $P$ an 'ordinary' member of the

\footnotetext{
${ }^{4}$ That a society could have just one member is not unreasonable as this may represent a player who chooses not to conform to the actions of similar players (Bernheim 1994).
} 
public. We suppose that there is only one celebrity. Members of the public like living in the same location as the celebrity. Thus, the payoff of a player with attribute $P$ is equal to 1 if he matches the choice of the celebrity and 0 otherwise. The celebrity, in contrast, prefers to avoid the public and thus his payoff is equal to the proportion of members of the public whose choice of location he mismatches. In any Nash equilibrium the celebrity must have probability $1 / 2$ of living in both locations. Thus, arbitrarily large games induced from this pregame need not have an approximate Nash equilibrium in pure strategies consistent with conformity since there may not exist an approximate Nash equilibrium in pure strategies.

Example 1 illustrates that some conditions will be required to guarantee the existence of an approximate Nash equilibrium in pure strategies. Our second example demonstrates that even if there exists an approximate Nash equilibrium in pure strategies there need not exist one that is consistent with conformity.

Example 2: Players choose between locations $A$ and $B$. The attribute space is $[0,1]$. A player's attribute determines whether he prefers location $A$ or $B$. Whether a player prefers $A$ or $B$ can, however, be seen as essentially a random event. More formally, assume that if a player has attribute $\omega$ where $\omega$ is a rational number then he is assigned a payoff of 1 for choosing $A$ and 0 for choosing $B$. If a player has attribute $\omega$ where $\omega$ is an irrational number then he is assigned a payoff of 1 for choosing $B$ and 0 for choosing $A$. Games induced from this pregame clearly have a Nash equilibrium in pure strategies. For arbitrarily large games, however, it is clear that no bound can be put on the number of societies that a Nash equilibrium would induce. For example, in a game where alternate players (in terms of the size of their attribute) have rational and irrational attributes the number of societies is as large as the player set.

Example 2 illustrates that some continuity assumption on attributes is necessary. In particular, we require that players with close attributes are similar. It would appear that a simple redefinition of an attribute would solve the observed problem with conformity in Example 2; for example, we could state that there are two attributes to represent those who like location $A$ and those who like location $B$. Note, however, that the attribute $\omega$ may signify an observable characteristic of a player that is irrelevant in terms of his payoff but does influence whether or not other players will identify with him; for example, $\omega$ may represent age and a player conforms to those with 
a similar age to himself. This suggests that conformity on the basis of $\omega$ may be observed, implying that $[0,1]$ is a relevant attribute space to consider.

\subsection{Large games}

To derive our main result we make two assumptions on pregames - continuity in attributes and global interaction. We introduce each in turn.

Continuity in Attributes: The pregame $\mathcal{G}=(\Omega, S, h)$ satisfies continuity in attributes if for any $\varepsilon>0$ there exists a real number $\delta_{c}(\varepsilon)>0$ such that, for any two games $\Gamma(N, \alpha)$ and $\Gamma(N, \bar{\alpha})$, if for all $i \in N$ it holds that

$$
\operatorname{dist}(\alpha(i), \bar{\alpha}(i))<\delta_{c}(\varepsilon)
$$

then for any $j \in N$ and for any pure strategy vector $m$,

$$
\left|u_{j}^{\alpha}(m)-u_{j}^{\bar{\alpha}}(m)\right|<\varepsilon .
$$

Continuity in attributes dictates that, given strategy choices, if the attribute function changes only slightly, then payoffs change only slightly. Note that Example 2 does not satisfy continuity in attributes.

To define global interaction we introduce a metric $\rho_{\alpha}$ on pure strategy vectors for a given game $\Gamma(N, \alpha)$. Consider two arbitrary pure strategy vectors $m, s \in S^{N}$ and denote by $w_{m}$ and $w_{s}$ the respective induced weight functions. Define $\rho_{\alpha}$ by

$$
\rho_{\alpha}(m, s) \stackrel{\text { def }}{=} \frac{1}{|N|} \sum_{s_{k} \in S} \sum_{\omega \in \alpha(N)}\left|w_{m}\left(\omega, s_{k}\right)-w_{s}\left(\omega, s_{k}\right)\right| .
$$

Thus, pure strategy vectors $m$ and $s$ are seen as 'close' if the proportion of players with each attribute playing each strategy is approximately the same.

Global Interaction: The pregame $\mathcal{G}=(\Omega, S, h)$ satisfies global interaction when for any $\varepsilon>0$ there exists a real number $\delta_{g}(\varepsilon)>0$ such that, for any game $\Gamma(N, \alpha)$ and any two pure strategy vectors $m$ and $s$, if

$$
\rho_{\alpha}(m, s)<\delta_{g}(\varepsilon)
$$

then for any $j \in N$ where $m_{j}=s_{j}$

$$
\left|u_{j}^{\alpha}(m)-u_{j}^{\alpha}(s)\right|<\varepsilon .
$$


The assumption of global interaction states that a player is nearly indifferent to small changes in the proportion, relative to the total population, of players of each attribute playing each strategy. ${ }^{5}$ Thus, the actions of any one player have little influence on the payoffs of others. We note that the pregame of Example 1 does not satisfy global interaction; when the number of ordinary people becomes large a change in the strategy of the celebrity has almost no effect on the metric $\rho_{\alpha}(m, s)$ but may have a large effect on the utility of ordinary people.

The pregame $\mathcal{G}=(\Omega, S, h)$ is said to satisfy the large game property if it satisfies both continuity in attributes and global interaction. The large game property implies a form of continuity of $h$ with respect to changes in the weight function and attribute. Indeed, to summarize: continuity in attributes is a bound on the payoff difference when the attributes of players change but their strategies do not. By contrast, global interaction is a bound on the payoff difference when the strategies of players change but their attributes do not. The pregame of Example 1, for instance, satisfies continuity in attributes but not global interaction. The pregame of Example 2 , by contrast, satisfies global interaction but not continuity in attributes.

\subsection{Purification}

The following result demonstrates that in sufficiently large games induced from a pregame satisfying the large game property there exists an approximate Nash equilibrium in pure strategies. This result is most easily obtained using a purification theorem due to Kalai (2000). ${ }^{6}$

Theorem 1: Consider a pregame $\mathcal{G}=(\Omega, S, h)$ that satisfies the large game property. Given any real number $\varepsilon>0$ there exists a real number $\eta(\varepsilon)$ such that for any population $(N, \alpha)$ where $|N|>\eta(\varepsilon)$ the induced game $\Gamma(N, \alpha)$ has a Nash $\varepsilon$-equilibrium in pure strategies. ${ }^{7}$

\footnotetext{
${ }^{5}$ With somewhat more restrictions on the model, the conditions of global interaction and continuity in attributes could be merged into one continuity condition. We prefer not to do this since we see global interaction as an important feature of the model. Also, the assumption of global interaction was motivated by assumptions on cooperative pregames that small groups of players have negligible impacts on large populations (cf., Wooders 1994a,b).

${ }^{6}$ The purification result of Kalai (2000) is sufficient for the purposes of the current paper. A general purification theorem, with countable action and type sets is presented in Cartwright and Wooders (2003b). Seminal results on purification by large numbers of players (an atomless continuum) appear in Schmeidler (1973) and Mas-Colell (1984). See Khan and Sun (2002) for a recent survey.

${ }^{7}$ It can easily be verified that $\eta(\varepsilon)$ is a function of $\varepsilon, K, \delta_{g}(\varepsilon)$ and $\delta_{c}(\varepsilon)$. Further, $\eta(\varepsilon)$
} 
Proof: Suppose not. Then there exists an $\varepsilon>0$ and, for every integer $\nu$, a game $\Gamma\left(N^{\nu}, \alpha^{\nu}\right)$ such that $\left|N^{\nu}\right|>\nu$ and game $\Gamma\left(N^{\nu}, \alpha^{\nu}\right)$ has no Nash $\varepsilon$-equilibrium. Let $\delta=\delta_{c}\left(\frac{\varepsilon}{3}\right)$ be the real number implied by continuity in attributes for a payoff bound of $\frac{\varepsilon}{3}$. Partition $\Omega$ into a finite number of subsets $\Omega_{1}, \ldots, \Omega_{Q}$ each of diameter less than $\delta$. For each $\Omega_{q}$ pick a point $\omega_{q} \in \Omega_{q}$. For each $\nu$ consider a population $\left(N^{\nu}, \bar{\alpha}^{\nu}\right)$ satisfying, for all $i \in N^{\nu}$, the property that $\bar{\alpha}^{\nu}(i)=\omega_{q}$ if and only if $\alpha^{\nu}(i) \in \Omega_{q}$. We note that, by the well known Nash existence theorem, each $\Gamma\left(N^{\nu}, \bar{\alpha}^{\nu}\right)$ has a Nash equilibrium. Consider the set of games $G=\left\{\Gamma\left(N^{\nu}, \bar{\alpha}\right)\right\}_{\nu}$. Given that the set of attributes for $G$ is finite, $G$ is a subset of a family of semi-anonymous Bayesian games as defined by Kalai (2000). From this, global interaction and the existence of Nash equilibrium, Theorem 1 of Kalai (2000) implies there exists $\nu^{*}$ such that any game $\Gamma\left(N^{\nu}, \bar{\alpha}^{\nu}\right)$ where $\nu>\nu^{*}$ has a Nash $\frac{\varepsilon}{3}$-equilibrium in pure strategies $m^{\nu}$. By continuity in attributes and the choice of $\delta$

$$
\left|u_{i}^{\alpha}\left(s_{k}, m_{-i}^{\nu}\right)-u_{i}^{\bar{\alpha}}\left(s_{k}, m_{-i}^{\nu}\right)\right|<\frac{\varepsilon}{3}
$$

for all $s_{k} \in S$. Thus, for $\nu>\nu^{*}, m^{\nu}$ is a Nash $\varepsilon$-equilibrium in pure strategies of game $\Gamma\left(N^{\nu}, \alpha^{\nu}\right)$.

\subsection{Main Result}

Theorem 2 demonstrates that in sufficiently large games there exists an approximate Nash equilibrium in pure strategies that partitions the population into a bounded number of societies. A fundamental aspect of Theorem 2 is that the bound is independent of population size. Note that the smaller is the bound the more dissimilar players in the same society may be. Theorem 2 is proved in an appendix.

Theorem 2: Let $\mathcal{G}=(\Omega, S, h)$ be a pregame satisfying the large game property. Given any real number $\varepsilon>0$ there exists real number $\eta(\varepsilon)$ and integer $A(\varepsilon)$ such that for any population $(N, \alpha)$ where $|N|>\eta(\varepsilon)$ the induced game $\Gamma(N, \alpha)$ has a Nash $\varepsilon$-equilibrium in pure strategies that induces a partition of the population $(N, \alpha)$ into $Q \leq A(\varepsilon) K$ societies. $^{8}$

is non-increasing in $\varepsilon, \delta_{g}(\varepsilon)$ and $\delta_{c}(\varepsilon)$. This is also the case for the population bound in Theorems 2 and 3.

${ }^{8}$ In fact a somewhat stronger result can be obtained: for any population $(N, \alpha)$ where $|N|>\eta(\varepsilon)$ and for any Nash equilibrium $\sigma$ of the induced game $\Gamma(N, \alpha)$, there exists a Nash $\varepsilon$-equilibrium in pure strategies $m$ where $\sigma$ and $m$ 'are close' and $m$ induces a partition of the population $(N, \alpha)$ into $Q \leq A(\varepsilon) K$ societies. 
Theorem 2 suggests that in sufficiently large games conformity can be individually rational. We highlight that the result applies to games in which all players have different attributes.

Note how the definitions of a society and of a partition of the population into societies leaves open the possibility that two players $i, j \in N$ with the same attributes could belong to different societies and play different strategies. A result such as Theorem 2 cannot be obtained unless this is permitted. ${ }^{9}$ To see this, consider a game $\Gamma(N, \alpha)$ where all players have the same attribute and any Nash equilibrium has the property that a positive fraction of the players choose one strategy and a positive fraction choose another strategy.

Another important feature of Theorem 2 is the convexity aspect of societies. For some attribute spaces, for example, $\Omega=\mathcal{C} \times[0,1]$, Theorem 2 implies the existence of an approximate Nash equilibria in pure strategies with the property that most players are playing the same strategy as their nearest neighbors in attribute space. This, however, is a special case; see Wooders, Cartwright and Selten (2001) for further discussion.

\subsection{Bounding the number of societies independently of $\varepsilon$}

In some cases it is possible to bound the number of societies independently of $\varepsilon$. We provide one such example.

For simplicity we shall assume that $F=1$ and the attribute space is thus $\Omega=\mathcal{C} \times[0,1]$. Further, we shall assume that if a player has attribute $(c, t)$ the value $c$ characterizes his external influence on others - his crowding type - while $t$ characterizes his payoff function - his taste type. We think of the crowding type of a player as such characteristics as gender, educational level, height, ability to salsa, and so on, that are observable to other players and may have direct effects on them. In contrast, we think of a player's taste type as of direct relevance only to himself, for example, whether he enjoys school or whether he likes to dance.

Two assumptions on crowding and taste types encapsulate these ideas. The first assumption, within type anonymity, implies that two players of the same crowding type, playing the same strategy, have the same influence on

\footnotetext{
${ }^{9}$ Wooders, Cartwright and Selten (2001) obtain a complementary result to Theorem 1 in which players with the same attribute do belong to the same society. This is possible by treating populations in which the number of players of any one attribute is bounded. Similarly the literature on non-atomic games demonstrates the existence of a symmetric Nash equilibrium in pure strategies in games with a continuum of players if the distribution of players over attributes is atomless (see Pascoa 1993).
} 
the payoffs of others.

Within (crowding) type anonymity: Pregame $\mathcal{G}=(\Omega, S, h)$ satisfies within type anonymity when for any induced game $\Gamma(N, \alpha)$ and for any two pure strategy vectors $m$ and $s$ if

$$
\sum_{\omega: \omega=(c, \cdot)} w_{m}\left(\omega, s_{k}\right)=\sum_{\omega: \omega=(c, \cdot)} w_{s}\left(\omega, s_{k}\right)
$$

for all $c \in \mathcal{C}$ then for any $i \in N$ where $m_{i}=s_{i}$

$$
u_{i}^{\alpha}\left(m_{i}, m_{-i}\right)=u_{i}^{\alpha}\left(s_{i}, s_{-i}\right) .
$$

That is, if any two strategy vectors have the property that, from the perspective of player $i$, the weight functions induced by these strategy vectors assign the same weight to each strategy chosen by players of each crowding type, then player $i$ is indifferent between the two situations.

Our second assumption is in the nature of Grandmont's (1978) 'intermediate preferences.' An explanation follows the definition.

Linearity in taste types: Pregame $\mathcal{G}=(\Omega, S, h)$ satisfies linearity in taste types if there exists a function $y: \mathcal{C} \times S \times W \rightarrow \mathbb{R}$ and a function $x: \mathcal{C} \times S \times W \rightarrow \mathbb{R}$ such that for any induced game $\Gamma(N, \alpha)$ and any pure strategy vector $m$ the payoff of player $i \in N$ where $\alpha(i)=(c, t)$ is given by

$$
u_{i}^{\alpha}\left(m_{i}, m_{-i}\right)=y\left(c, m_{i}, w_{m}\right)+t \cdot x\left(c, m_{i}, w_{m}\right) .
$$

We recall that a player's taste type is determined by $t \in[0,1]$. All else equal, if a player's taste type is a convex combination of the taste types of two other players (and all three players have the same crowding type), then his payoff is the same convex combination of the payoffs of the other two players. Intuitively, we could think of there being a 'representative player' for each crowding type with, say, taste type 0.5. The payoff of a player with taste type $t$ can then be thought of as a linear function of how much his taste type differs from that of the representative for his crowding type.

In Section 3.5 we consider a pregame that satisfies within type anonymity and linearity in taste types. Our second main result, Theorem 3, places a bound that is independent of $\varepsilon$ on the number of societies. For simplicity we state and prove Theorem 3 for $K=2$ and $F=1$; in two footnotes in the proof we indicate how these two restrictions can be relaxed. 
Theorem 3: Let $\mathcal{G}=(\Omega, S, h)$ be a pregame satisfying the large game property, within type anonymity and linearity in taste types. Let $K=2$ and $F=1$. Given any real number $\varepsilon>0$ there exists real number $\eta(\varepsilon)$ such that for any population $(N, \alpha)$ where $|N|>\eta(\varepsilon)$ the induced game $\Gamma(N, \alpha)$ has a Nash $\varepsilon$-equilibrium in pure strategies that induces a partition of the population $(N, \alpha)$ into $Q \leq 2 C$ societies.

Proof: By Theorem 1 for any sufficiently large population $(N, \alpha)$ the induced game $\Gamma(N, \alpha)$ has a Nash $\varepsilon$-equilibrium in pure strategies $m^{*}$. Let $M$ denote the set of pure strategy vectors such that $m \in M$ if and only if $m$ is a Nash $\varepsilon$-equilibrium and

$$
\sum_{\omega: \omega=(c, \cdot)} w_{m^{*}}\left(\omega, s_{k}\right)=\sum_{\omega: \omega=(c, \cdot)} w_{m}\left(\omega, s_{k}\right)
$$

for all $c$ and $s_{k}$. We note that $m^{*} \in M$ and so $M$ is non-empty.

Consider a pure strategy vector $m \in M$. For each $c$ and $s_{k}$ let $T_{c k} \subset[0,1]$ be such that $t \in T_{c k}$ if and only if there exists a player $i \in N$ satisfying $\alpha(i)=(c, t)$ and $m_{i}=s_{k}$. Let $X_{c k} \subset N$ be such that $j \in X_{c k}$ if and only if $\alpha(j)=(c, t)$ where $t \in \operatorname{int}\left(\operatorname{con}\left(T_{c k}\right)\right)$ and $m_{j} \neq s_{k} \cdot{ }^{10}$ Let $X=\cup_{c, k} X_{c k}$. If $|X|=0$ then $m$ induces a partition of the population into $2 C$ societies. Suppose $|X|>0$. We will construct from $m$ a pure strategy vector $m^{i} \in M$ that diminishes $|X|$ by one. Iterating this argument one obtains an $\bar{m} \in M$ giving $|X|=0$ thus proving the result.

Given that $|X|>0$, we can select a nonempty set $X_{c k}$ for some $c$ and $s_{k}$ and a player $j \in X_{c k}$. Thus, $\alpha(j)=(c, t)$ and $t \in \operatorname{int}\left(\operatorname{con}\left(T_{c k}\right)\right)$ yet $m_{j} \neq s_{k}$. Assume that $m_{j}=s_{\bar{k}}$. Let $A_{c k} \subset N$ be such that $i \in A_{c k}$ where $\alpha(i)=(c, t)$ if and only if $m_{i}=s_{k}$ and $t$ belongs to the boundary of $\operatorname{con}\left(T_{c k}\right) .{ }^{11}$ For each $i \in A_{c k}$ let $m^{i}$ be the pure strategy vector with the properties that $m_{i}^{i}=s_{\bar{k}}, m_{j}^{i}=s_{k}$ and $m_{l}^{i}=m_{l}$ for all other $l \in N$; thus players $i$ and $j$ have 'exchanged' pure strategies. We conjecture $\left(^{*}\right)$ that for some $i^{*} \in A_{c k}$ the pure strategy vector $m^{i^{*}}$ is a Nash $\varepsilon$-equilibrium. Provided this conjecture holds, given that $i^{*}$ belongs to the boundary of $\operatorname{con}\left(T_{c k}\right)$ and $K=2$ the value of $|X|$ is one less for $m^{i^{*}}$ then for $m .^{12}$

\footnotetext{
${ }^{10}$ Note that $X_{c k}$ may be the empty set. For example, if every player of crowding type $c$ has the same taste type $t$ then $X_{c k}$ will be empty for all $s_{k}$.

${ }^{11}$ There must be some such player since the convex hull $\operatorname{int}\left(\operatorname{con}\left(T_{c k}\right)\right)$ is determined by players in $A_{c k}$ and $\operatorname{int}\left(\operatorname{con}\left(T_{c k}\right)\right) \neq \varnothing$.

${ }^{12}$ If $K=2$ and $F>1$ then this argument no longer suffices. Further 'exchanges' of strategies may be necessary to reduce $|X|$ by one.
} 
To prove the conjecture $(*)$ observe that within type anonymity and linearity in taste types implies that for some $\beta_{1}, \beta_{2}, \ldots, \beta_{\left|A_{c k}\right|}$ [where $1 \geq$ $\beta_{i} \geq 0$ and $\left.\sum \beta_{i}=1\right]$

$$
u_{j}^{\alpha}\left(z, m_{-j}\right)=\sum_{i \in A_{c k}} \beta_{i} u_{i}^{\alpha}\left(z, m_{-i}^{i}\right)
$$

for all $z \in S$. Given that $m$ is a Nash $\varepsilon$-equilibrium

$$
u_{j}^{\alpha}\left(s_{\bar{k}}, m_{-j}\right) \geq u_{j}^{\alpha}\left(s_{k}, m_{-j}\right)-\varepsilon .
$$

Thus, there exists some $i^{*} \in A_{c k}$ and corresponding $m^{i^{*}}$ where ${ }^{13}$

$$
u_{i^{*}}^{\alpha}\left(m_{-i^{*}}^{i^{*}}, m_{-i^{*}}^{i^{*}}\right) \geq u_{i^{*}}^{\alpha}\left(s_{k}, m_{-i^{*}}^{i^{*}}\right)-\varepsilon .
$$

It is clear, by within type anonymity, that

$$
u_{l}^{\alpha}\left(m_{l}^{i^{*}}, m_{-l}^{i^{*}}\right) \geq u_{l}^{\alpha}\left(z, m_{-l}^{i^{*}}\right)-\varepsilon
$$

for all $z \in S$ and $l \in N, l \neq i^{*}, j$. It thus remains to consider player $j$. Let $l \in A_{c k}$ and $l \neq i^{*}$. Within type anonymity and linearity of taste types implies that

$$
u_{j}^{\alpha}\left(z, m_{-j}^{i^{*}}\right)=\sum_{i \in A_{c k} \backslash i^{*}} \beta_{i} u_{i}^{\alpha}\left(z, m_{-i}^{i^{*}}\right)+\beta_{i^{*}} u_{i^{*}}^{\alpha}\left(z, m_{-i^{*}}^{l}\right)
$$

for all $z \in S$. Thus,

$$
u_{j}^{\alpha}\left(m_{j}^{i^{*}}, m_{-j}^{i^{*}}\right) \geq u_{j}^{\alpha}\left(s_{\bar{k}}, m_{-j}^{i^{*}}\right)-\varepsilon
$$

and $m^{i^{*}}$ is a Nash $\varepsilon$-equilibrium.

\section{Conclusions}

If individuals tend to conform and to be influenced by the actions of others then this poses a challenge to the individual rationality assumption of game theory. This challenge leads us to question the possible existence of an approximate Nash equilibrium consistent with conformity. In this paper we

\footnotetext{
${ }^{13}$ If $K>2$, while this inequality holds, it would no longer be sufficient to demonstrate that playing $m_{-i^{*}}^{i^{*}}$ is an $\varepsilon$-best response for player $i^{*}$. In particular, if $z$ is a third strategy then we may have, $u_{i^{*}}^{\alpha}\left(m_{-i^{*}}^{i^{*}}, m_{-i^{*}}^{i^{*}}\right)<u_{i^{*}}^{\alpha}\left(z, m_{-i^{*}}^{i^{*}}\right)-\varepsilon$. For the case of $K=3$ this can be overcome by commencing with a Nash $\frac{\varepsilon}{2}$-equilibrium.
} 
introduce a notion of societies that allows us to demonstrate the existence of such an equilibrium in games with many players.

Issues that still remain include: (i). We only demonstrate the existence of an approximate Nash equilibrium with conformity; we do not address directly, the question of whether players actually learn to play that equilibrium. This is discussed by Cartwright (2003) where sufficient conditions are provided under which play will indeed converge to the desired equilibrium. (ii). Conformity in mixed strategy equilibrium is not treated. To treat conformity in mixed strategies may seem unmotivated given our insistence on pure strategy equilibria. Note, however, that while it may seem unnatural that a player would use a mixed strategy it need not be unnatural that a society would 'play a mixed strategy'. In Cartwright and Wooders (2003a) we consider this possibility by formulating conformity in terms of mixed strategies and incomplete information.

\section{Appendix}

Lemma 1: Let $\mathcal{G}=(\Omega, S, h)$ be a pregame satisfying the large game property. For any induced game $\Gamma(N, \alpha)$, for any partition of $\Omega$ into a finite number of subsets $\Omega_{1}, \ldots, \Omega_{A}$, each of diameter less than $\delta_{c}\left(\frac{\varepsilon}{3}\right)$, and for any two pure strategy vectors $m$ and $\bar{m}$ where

$$
\sum_{\omega \in \Omega_{a}} w_{\alpha, m}\left(\omega, s_{k}\right)=\sum_{\omega \in \Omega_{a}} w_{\alpha, \bar{m}}\left(\omega, s_{k}\right)
$$

if $m$ is a Nash $\frac{\varepsilon}{3}$-equilibrium in pure strategies then $\bar{m}$ is a Nash $\varepsilon$-equilibrium in pure strategies. ${ }^{14}$

Proof: Given an induced game $(N, \alpha)$ and two pure strategy vectors $m$ and $\bar{m}$ satisfying (4), it is immediate that there exists a one-to-one mapping $R(i): N \rightarrow N$ such that,

$$
\bar{m}_{i}=m_{R(i)}
$$

for all $i \in N$ and,

$$
\operatorname{dist}(\alpha(i), \alpha(R(i)))<\delta_{c}\left(\frac{\varepsilon}{3}\right)
$$

Informally, we can treat equivalently: (a) player $i$ having attribute $\omega=\alpha(i)$ and playing strategy $m_{R(i)}$ and (b) player $R(i)$ playing strategy $m_{R(i)}$ and

\footnotetext{
${ }^{14}$ Where $\delta_{c}\left(\frac{\varepsilon}{3}\right)$ is the real number implied by continuity in attributes for a payoff bound of $\frac{\varepsilon}{3}$.
} 
having attribute $\omega=\alpha(i)$. Thus, consider the population $(N, \bar{\alpha})$ where,

$$
\bar{\alpha}(R(i))=\alpha(i)
$$

for all $i \in N$. Our method of proof is to (i) demonstrate that $m$ is a Nash $\varepsilon$-equilibrium in pure strategies of game $\Gamma(N, \bar{\alpha})$ before (ii) demonstrating how this implies that $\bar{m}$ is a Nash $\varepsilon$-equilibrium in pure strategies of game $\Gamma(N, \alpha)$.

The assumption of continuity in attributes and (6) implies,

$$
\left|u_{i}^{\alpha}\left(s_{k}, m_{-i}\right)-u_{i}^{\bar{\alpha}}\left(s_{k}, m_{-i}\right)\right|<\frac{\varepsilon}{3}
$$

for all $s_{k} \in S$ and all $i \in N$. Given that $m$ is a Nash $\frac{\varepsilon}{3}$-equilibrium in pure strategies for $\Gamma(N, \alpha)$ it follows that

$$
u_{i}^{\alpha}\left(m_{i}, m_{-i}\right) \geq u_{i}^{\alpha}\left(s_{k}, m_{-i}\right)-\frac{\varepsilon}{3} .
$$

The above two inequalities yield

$$
\begin{gathered}
u_{i}^{\bar{\alpha}}\left(m_{i}, m_{-i}\right) \geq u_{i}^{\alpha}\left(m_{i}, m_{-i}\right)-\frac{\varepsilon}{3} \\
\geq u_{i}^{\alpha}\left(s_{k}, m_{-i}\right)-\frac{2 \varepsilon}{3} \\
\geq u_{i}^{\bar{\alpha}}\left(s_{k}, m_{-i}\right)-\varepsilon
\end{gathered}
$$

for all $i \in N$ and $s_{k} \in S$. Thus, $m$ is a Nash $\varepsilon$-equilibrium in pure strategies of game $\Gamma(N, \bar{\alpha})$.

By (5) and (7)

$$
\left(\bar{\alpha}(R(i)), m_{R(i)}\right)=\left(\alpha(i), m_{R(i)}\right)=\left(\alpha(i), \bar{m}_{i}\right)
$$

for all $i \in N$. It follows that

$$
u_{R(i)}^{\bar{\alpha}}\left(s_{k}, m_{-R(i)}\right)=u_{i}^{\alpha}\left(s_{k}, \bar{m}_{-i}\right)
$$

for all $i \in N$ and all $s_{k} \in S$. It is immediate from (9) and (11) that $\bar{m}$ is a Nash $\varepsilon$-equilibrium in pure strategies of game $\Gamma(N, \alpha)$.

We recall that $\Omega=\{1,2, \ldots, C\} \times[0,1]^{F}$ for some finite integers $C$ and $F$. We make use of a lexicographic ordering on elements of $[0,1]^{F}$. Formally, we define the binary relations $<_{L}$ and $=_{L}$ as follows: Take any two points $t=\left(t_{1}, \ldots, t_{F}\right), \tau=\left(\tau_{1}, \ldots, \tau_{F}\right) \in[0,1]^{F}$. We say that $t={ }_{L} \tau$ if and only if $t_{f}=\tau_{f}$ for all $f=1, \ldots, F$. We say that $t<_{L} \tau$ if either:

$$
\text { 1. } \sum_{f} t_{f}<\sum_{f} \tau_{f} \text { or, }
$$


2. $\sum_{f<f^{*}} t_{f}=\sum_{f} \tau_{f}$ and for some $f^{*}$ we have $t_{f^{*}}<\tau_{f^{*}}$ and $t_{f}=\tau_{f}$ for all $f<f^{*}$.

We say that $t \leq_{L} \tau$ if either $t<_{L} \tau$ or $t={ }_{L} \tau$.

Lemma 2: Given any two finite sets of points $\Omega_{J}=\left\{t^{1}, \ldots, t^{J}\right\}$ and $\Omega_{Q}=$ $\left\{\tau^{1}, \ldots, \tau^{Q}\right\}$ (where $t^{1}, \ldots, t^{J}, \tau^{1}, \ldots, \tau^{Q} \in[0,1]^{F}$ ) if $t^{j} \leq_{L} \tau^{q}$ for all $j$ and $q$ then the interior of the convex hulls of $\Omega_{J}$ and $\Omega_{Q}$ are either distinct or both empty.

Proof: Suppose the claim is false. Then there exists a point $\omega \in \Omega$ such that $\omega \in I\left(C o\left(\Omega_{J}\right)\right)$ and $\omega \in I\left(C o\left(\Omega_{Q}\right)\right)$. Thus, for non-negative numbers $\gamma_{1}, \ldots, \gamma_{J}$ and $\beta_{1}, \ldots, \beta_{Q}$

$$
\sum_{q} \beta_{q}=\sum_{j} \gamma_{j}=1
$$

and, for each $f=1, \ldots, F$,

$$
\omega_{f}=\sum_{j} \gamma_{j} t_{f}^{j}=\sum_{q} \beta_{q} \tau_{f}^{q} .
$$

This implies that

$$
\sum_{j}\left[\gamma_{j} \sum_{f} t_{f}^{j}\right]=\sum_{q}\left[\beta_{q} \sum_{f} \tau_{f}^{q}\right]
$$

By assumption, for each $t^{j} \in \Omega_{J}$ and $t^{q} \in \Omega_{Q}$ it holds that $\sum_{f} t_{f}^{j} \leq$ $\sum_{f} t_{f}^{q}$. Suppose, for some $t^{\bar{j}} \in \Omega_{J}$ and $\tau^{\bar{q}} \in \Omega_{Q}$, that $\sum_{f} t_{f}^{\bar{j}}<\sum_{f} \tau_{f}^{\bar{q}}$. Given (14) it must be that either $\gamma_{\bar{j}}=0$ or $\beta_{\bar{q}}=0$. Let $\Omega_{J}^{+}$denote the set of $t^{j} \in \Omega_{J}$ given positive weight $\gamma_{j}>0$ and $\Omega_{Q}^{+}$the set of all $\tau^{q} \in \Omega_{Q}$ given positive weight $\beta_{q}>0$. It is immediate that $\sum_{f} t_{f}^{j}=\sum_{f} \tau_{f}^{q}$ for each $t^{j} \in$ $\Omega_{J}^{+}$and $\tau^{q} \in \Omega_{Q}^{+}$.

If $\Omega_{J}^{+}=\Omega_{Q}^{+}$then we easily obtain the desired contradiction. When $\Omega_{J}^{+}=$ $\Omega_{Q}^{+}$and for each element $t^{j}$ in $\Omega_{J}^{+}$it holds that $t^{j} \leq_{L} t^{q}$ then the sets must each contain only one element and, in this case, the interiors of the convex hulls are both empty.

Let $\Omega_{J}^{++}=\Omega_{J}^{+} \backslash \Omega_{Q}^{+}$and $\Omega_{Q}^{++}=\Omega_{Q}^{+} \backslash \Omega_{J}^{+}$. Either $\Omega_{J}^{++}$or $\Omega_{Q}^{++}$is nonempty. Suppose that $\Omega_{J}^{++}$is non-empty. For every $t^{j} \in \Omega_{J}^{++}$and $\tau^{q} \in \Omega_{Q}^{+}$ 
there is some $f^{*} \in\{1, \ldots, F-1\}$ for which $t_{f^{*}}^{j}<\tau_{f^{*}}^{q}$ and $t_{f}^{j}=\tau_{f}^{q}$ for all $f<f^{*}$. Take the minimum of these $f^{*}$ over all points $t^{j} \in \Omega_{J}^{++}$and $\tau^{q} \in$ $\Omega_{Q}^{+}$. By choice of $f^{*}$ it holds that $t_{f^{*}}^{j} \leq \tau_{f^{*}}^{q}$ for all $j \in \Omega_{J}^{+}$and $q \in \Omega_{Q}^{+}$and $t_{f^{*}}^{\bar{j}}<\tau_{f^{*}}^{\bar{q}}$ for some $\bar{j} \in \Omega_{J}^{++}$and $\bar{q} \in \Omega_{Q}^{+}$. This must contradict either (12) or (13). The case where $\Omega_{Q}^{++}$is non-empty can be treated in an analogous manner.

Proof of Theorem 2: Consider some $\varepsilon>0$ and set $\delta=\delta_{c}\left(\frac{\varepsilon_{0}}{3}\right)>0$, where $\delta_{c}\left(\frac{\varepsilon_{0}}{3}\right)$ is the real number implied by continuity in attributes for a payoff bound of $\frac{\varepsilon_{0}}{3}$. Use compactness of $\Omega$ to write $\Omega$ as the disjoint union of a finite number $A$ of convex non-empty subsets $\Omega_{1}, \ldots, \Omega_{A}$, each of diameter less than $\delta .{ }^{15}$ We claim that $A$ satisfies the conditions required by the Theorem. By Theorem 1 there exists real number $\eta\left(\frac{\varepsilon}{3}\right)$ such that every game $\Gamma(N, \alpha)$ satisfying $|N|>\eta\left(\frac{\varepsilon}{3}\right)$ has a Nash $\frac{\varepsilon}{3}$ equilibrium in pure strategies.

Let $\Gamma(N, \alpha)$ be any game where $|N|>\eta\left(\frac{\varepsilon}{3}\right)$ and let $m$ denote a Nash $\frac{\varepsilon}{3}$ equilibrium in pure strategies. Consider a change of pure strategy vector from $m$ to $\bar{m}$ where $\bar{m}$ satisfies:

1. for all $\Omega_{a}$ and $s_{k} \in S$,

$$
\sum_{\omega \in \Omega_{a}} w_{\alpha, m}\left(\omega, s_{k}\right)=\sum_{\omega \in \Omega_{a}} w_{\alpha, \bar{m}}\left(\omega, s_{k}\right)
$$

2. for any $i, j \in N$ where $\alpha(i), \alpha(j) \in \Omega_{a}$ for some $a$, if $m_{i}=s_{k}$ and $m_{j}=s_{\bar{k}}$ where $k<\bar{k}$ then $\alpha(i) \leq \alpha(j)$.

Given that the finite set of points $\alpha(N)$ is well ordered, it is always possible to construct such a pure strategy vector $\bar{m}$ by a simple 'reassignment' of pure strategies. Given the choice of $\delta$ and that $m$ is a Nash $\frac{\varepsilon}{3}$-equilibrium in pure strategies it is immediate from Lemma 1 that $\bar{m}$ is a Nash $\varepsilon$-equilibrium in pure strategies. By applying Lemma 2 and recalling that each $\Omega_{a}$ is convex it is clear that $\bar{m}$ is a Nash $\varepsilon$-equilibrium in pure strategies that induces a partition of the population into at most $A K$ societies.

\section{References}

[1] Cartwright, E. (2003) "Learning to play approximate Nash equilibria in games with many players," The Warwick Economic Research Paper Series no. 671. On-line at http://www2.warwick.ac.uk/fac/soc/economics/research/papers/.

\footnotetext{
${ }^{15}$ Note that $A$ depends on $\varepsilon$ through its dependence on $\delta$.
} 
[2] Cartwright, E. and M. Wooders (2003a) "Social conformity in arbitrary games with incomplete information," The Warwick Economic Research Paper Series no. 672. On-line at http://www2.warwick.ac.uk/fac/soc/economics/research/papers/.

[3] Cartwright, E. and M. Wooders (2003b) "On purification of mixed strategy equilibria in games with incomplete information," The Warwick Economic Research Paper Series no. 686.

[4] Conley, J. and M.H. Wooders (2001) "Tiebout economics with differential genetic types and endogenously chosen crowding characteristics," Journal of Economic Theory 98, 261-294.

[5] Grandmont, J.M. (1978) "Intermediate preferences and the majority rule," Econometrica 46, 317-330.

[6] Kalai, E. (2000) "Private information in large games," Northwestern University Discussion Paper 1312 (November 2000), on-line at http://www.kellogg.northwestern.edu/research/math/dp_list_4.htm.

[7] Khan, A. and Y. Sun (2002) "Noncooperative games with many players," Handbook of Game Theory, R. Auman and S. Hart, eds. North Holland.

[8] Mas-Colell, A. (1984) "On a theorem of Schmeidler," Journal of Mathematical Economics 13: 206-210.

[9] Pascoa, M. (1993) "Approximate equilibrium in pure strategies for nonatomic games," Journal of Mathematical Economics 22: 223-241.

[10] Schmeidler, D. (1973) "Equilibrium points of nonatomic games," Journal of Statistical Physics 7: 295-300.

[11] Wooders, M., E. Cartwright and R. Selten (2001) "Some first results for noncooperative pregames: Social conformity and equilibrium pure strategies," (May 2001, revised August 2001 ), The Warwick Economic Research Paper Series no. 589, on-line at http://www.myrnawooders.com. 